\title{
Humoral response to a 13-valent pneumococcal conjugate vaccine in kidney
}

transplant recipients

Simon Heckel ${ }^{1,2,3}$, Monika Lindemann ${ }^{3}$, David Goldblatt ${ }^{4,5}$, Polly Burbidge ${ }^{4,5}$, Peter A. Horn $^{3}$, Benjamin Wilde ${ }^{2}$, Oliver Witzke ${ }^{1}$

To Authors' names:

${ }^{1}$ Department of Infectious Diseases, ${ }^{2}$ Department of Nephrology and ${ }^{3}$ Institute for Transfusion Medicine, University Hospital Essen, University Duisburg-Essen, Essen, Germany

${ }^{4}$ University College London Institute of Child Health, London, United Kingdom ${ }^{5}$ World Health Organisation, Pneumococcal Serology Reference Laboratory, London, United Kingdom

Address correspondence to: Monika Lindemann, M.D., Institute for Transfusion Medicine, University Hospital Essen, Virchowstraße 179, 45147 Essen, Germany. E-mail: monika.lindemann@uk-essen.de

Abstract word count: 252

Total text word count: 4300

Total number of Figures/Tables: $\quad 3 / 3$

Keywords: Pneumococcal conjugate vaccine, PCV13, kidney transplantation, OPA, serotype specific pneumococcal assay, global pneumococcal assay 


\section{Support}

This study was supported by Vaccine Research, Pfizer Inc., Pearl River, New York, USA, performing measurement of the OPA titers.

\section{Contributions}

Conception and design: Oliver Witzke, Monika Lindemann, Benjamin Wilde, Simon Heckel

Provision of study materials and patients: Oliver Witzke

Collection and assembly of data: Simon Heckel, Monika Lindemann, Oliver Witzke,

David Goldblatt, Polly Burbidge

Data analysis and interpretation: Simon Heckel, Monika Lindemann, Benjamin Wilde, Oliver Witzke

Manuscript writing: Simon Heckel, Monika Lindemann, David Goldblatt, Peter A. Horn, Oliver Witzke

\section{Conflict of interest disclosure}

O. W. has received consultancy and speaker's fees from Novartis Pharma, Roche AG, Pfizer, Wyeth and Astellas. The remaining authors indicate no potential conflict of interest.

\section{Ethics}

The study was approved by the local ethics committee (Nr. 14-5858-BO). All patients provided written informed consent. 


\section{Abbreviations}

CKD-EPI, Chronic Kidney Disease Epidemiology Collaboration; ELISA, enzyme linked immuosorbent assay; eGFR, estimated glomerular filtration rate; GMC, Geometric mean concentration; GMCR, geometric mean concentration ratio; GMT, geometric mean titer; GMTR, geometric mean titer ratio; HA, healthy adults of the reference populations; Ig, immunoglobulin; IPD, invasive pneumococcal disease; LLOQ, lower limit of quantification; KTR, kidney transplant recipients; MMF, mycophenolate mofetil; OPA, opsonophagocytic assay; PCP, pneumococcal capsular polysaccharide; PCV7, 7-valent pneumococcal conjugate vaccine; PCV13, 13-valent pneumococcal conjugate vaccine; S. pneumoniae, Streptococcus pneumoniae. 


\section{Abstract}

Introduction and Background. Vaccination against S. pneumoniae is recommended by national guidelines. Moderate immunogenicity of the 7 - and 13-valent pneumococcal conjugate vaccine (PCV7/13) has been reported in adult kidney transplant recipients (KTR). This study aims to further define the immunogenicity of PCV13 in this cohort.

Materials and Methods. 49 KTR were immunized with PCV13. A validated opsonophagocytic killing assay (OPA), a global anti-pneumococcal capsular polysaccharide (anti-PCP) IgG, IgG2, IgM and IgA ELISA, and - for selected patients a serotype specific anti-PCP WHO reference ELISA were performed prevaccination and at month 1 and 12 postvaccination.

Results. Geometric mean OPA titers increased significantly for $13 / 13$ serotypes at month 1 and for 10/13 serotypes at month 12 postvaccination. Vaccine response defined as an OPA titer $\geq 1: 8$ was reached in $9 / 13$ serotypes (median). $53 \%$ reached the vaccine response criteria at month 1 and $45 \%$ at month 12 . At month 1 after vaccination, the median OPA titer in an age-matched healthy reference population was 5- to 10-fold higher than in KTR. OPA titers correlated strongly with results to the global and serotype specific anti-PCP IgG ELISA. Decreased OPA titers significantly $(p<0.05)$ correlated with albuminuria, an interval between vaccination and transplantation $<12$ months, age and treatment with mycophenolate mofetil. Global IgG, $\lg G 2$, IgM and $\operatorname{IgA}$, as well as serotype specific anti-PCP antibody concentrations (12/13 serotypes) increased significantly at month 1 and 12 postvaccination.

Conclusions: Kidney transplant recipients show a significant humoral response after vaccination with PCV13. Functional antibody response, however, is reduced as compared to healthy adults. 


\section{Keywords}

Pneumococcal conjugate vaccine; opsonophagocytic assay; kidney transplant recipients, serotype specific; pneumococcal antibody global serum assays; pneumococcal vaccination in immunocompromised individuals

\section{Introduction}

Infectious complications are a major cause of morbidity and mortality in high risk populations such as solid organ recipients ${ }^{1}$. Lower respiratory tract infections (LRTI) are leading infectious complications after transplantation ${ }^{2}$. S. pneumoniae is a gram positive capsulated bacterium that continues to be the most commonly identified bacterial agent of pneumonia ${ }^{3}$. The use of immunosuppressive drugs increases the risk of invasive pneumococcal disease (IPD) up to 2.7 fold $^{4}$. An increase of antibiotic resistant strains emphasizes the need for vaccine induced protection ${ }^{5}$. Since June 2017,141 countries worldwide have introduced pneumococcal conjugate vaccines into the infant immunization programmes ${ }^{6}$ with reduction in invasive pneumococcal disease in both the immunized and unimmunized population ${ }^{7}$. The proportion of IPD-cases in adults caused by PCV13 serotypes in Germany 2017 has reduced to $30 \%$ as a consequence of universal infant immunization with PCV13\% ${ }^{8}$. In Germany a sequential administration of the 13-valent pneumococcal conjugate vaccine (PCV13) followed by the 23-valent pneumococcal polysaccharide vaccine (PPSV23) after 6-12 months is recommended for immunocompromised individuals ${ }^{9}$. The protection afforded by this vaccine regimen is unclear $^{10}$. PCV7, PCV13 and PPSV23 have been declared safe and immunogenic in kidney transplant recipients $(K T R)^{10-17}$. The immunogenicity of PCV7 has been shown to be non-inferior to that of PPSV23, but no enhanced immunogenicity was reported in kidney transplant recipients ${ }^{14}$. Increased serotype specific anti-PCP antibody concentrations for all serotypes and increased functional 
response for a preselected subset of serotypes induced by PCV13 have recently been demonstrated in $\mathrm{KTR}^{12}$.

The objective of this study was to evaluate the functional serological response to PCV13 and to correlate the functional response with anti-pneumococcal capsular polysaccharide (anti-PCP) antibody concentrations in KTR in order to inform future decisions about the optimal use of PCV's in the setting of KTR and to explore factors influencing vaccine immunogenicity.

\section{Results}

\subsection{Serotype specific pneumococcal opsonophagocytic assay}

The opsonophagocytic assay (OPA) was performed for 13 serotypes in 145 serum samples (49 prevaccination, 49 at month 1 and 47 at month 12 postvaccination). $10.5 \%$ of the values were either indeterminable or the quantity of the serum was not sufficient for assessment.

Vaccine response criteria defined as a positive OPA titer for at least 9 of 13 serotypes $(\approx 70 \%)^{18}$ were met by 4 of $49(8 \%)$ patients at prevaccination, 26 of $49(53 \%)$ patients at month 1 and 21 of $47(45 \%)$ at month 12 postvaccination. For each serotype, an OPA titer $\geq 1: 8$ was measurable in median $35 \%$ (range: $18-50 \%$ ) of patients prevaccination which increased to $76 \%(55-85 \%)$ at month 1 and $61 \%(38-74 \%)$ at month 12 postvaccination.

Comparing month 1 and prevaccination OPA titers for each serotype, in the median $41 \%$ (range: $32-58 \%$ ) of patients responded with a fold increase $\geq 4$; and $29 \%$ (1247\%) comparing month 12 and prevaccination date. 
Comparing month 1 and prevaccination geometric mean titers (GMTs), a significant increase was observed for all serotypes. Comparing the difference between month 12 and prevaccination GMTs, patients still had significantly elevated GMTs at month 12 for all serotypes except for serotype 14 and 23F. The lowest GMT and lowest increase were seen for serotype 3 (month 1 and 12). The highest GMT was observed for serotype $6 \mathrm{~B}$. The highest increase from baseline was reached for serotype $6 \mathrm{~A}$ (month 1) and 6B (month 12).

In Figure 1 and Suppl. Tab 1, GMTs and geometric mean titer ratios (GMTR) of serotype specific anti-PCP IgG for each serotype are presented.

Comparing the median GMTR of a healthy historical adult PCV vaccinated reference group and KTR, KTR aged 18-49, 50-59 or $\geq 60$ years showed a significantly decreased vaccine response ${ }^{19,20}$ (Tab. 2).

In detail (Suppl. Tab 2), with a median GMTR (HA/KTR) of 0.7 (range: 0.04-1.6), patients between 18 and 49 years exhibited higher prevaccination GMTs than a healthy reference group. But this difference was not significant. At month 1 and 12 postvaccination, KTR had significantly lower GMTs with a median GMTR of 4.8 (1.78.9) at month 1 and $2.6(1.1-4.6)$ at month 12. KTR aged 50-59 displayed significantly lower GMTs than healthy adults at month 1 postvaccination, with a median GMTR of 10.0 (2.8-48.0). KTR aged $\geq 60$ had prevaccination GMTs comparable to healthy adults, with a median GMTR of 1.1 (0.7-3.2). GMTs at month 1 and 12 , however, were significantly lower in KTR, with a median GMTR of 10.1 (2.6-34.0) at month 1 and 5.2 (2.6-18.2) at month 12 postvaccination. 
Serotype specific anti-PCP $\lg \mathrm{I} \geq 0.35$ and $\mathrm{OPA} \geq 1: 8$ were observed for 6 serotypes prevaccination (median), which increased to 9 at month 1 and at month 12 postvaccination.

\subsection{Global pneumococcal ELISA}

The global lgG, IgG2, IgM and IgA ELISA was performed for 23 serotypes in 145 serum samples (49 prevaccination, 49 at month 1 and 47 at month 12 postvaccination) (Fig. 2 and Suppl. Tab. 3).

The geometric mean concentration (GMC) of $\lg G$ antibodies against 23 serotypes increased from $29.7 \mathrm{mg} / \mathrm{l}$ prevaccination to $56.5 \mathrm{mg} / \mathrm{l}(\mathrm{p}<0.0001)$ at month 1 and 43.9 $\mathrm{mg} / \mathrm{l}(\mathrm{p}=0.003)$ at month 12 postvaccination. Compared to $\mathrm{GMC}$ in a healthy unimmunized adult reference group, the prevaccination IgG geometric mean concentration ratio (GMCR) was 0.7 (HA/KTR), which increased to 1.3 at month 1 and 1.0 at month 12 postvaccination. At prevaccination, $36.7 \%$ of patients had global IgG antibody concentrations comparable to the reference group (defined as $\geq 43.8 \mathrm{mg} / \mathrm{l}^{21}$ ) which increased to $69.4 \%$ at month 1 and $61.7 \%$ at month 12 postvaccination.

The GMC of IgG2 antibodies increased from $13.8 \mathrm{mg} / \mathrm{l}$ prevaccination to $30.8 \mathrm{mg} / \mathrm{l}$ $(p<0.0001)$ at month 1 and $23.2 \mathrm{mg} / \mathrm{l}(\mathrm{p}<0.0001)$ at month 12 postvaccination. Compared to GMC in the reference group, prevaccination IgG2 GMCR was 0.7, which increased to 1.5 at month 1 and 1.1 at month 12 postvaccination. At prevaccination date, $30.6 \%$ of patients had global lgG2 antibody concentrations comparable to the reference group (defined as $\geq 20.5 \mathrm{mg} / \mathrm{l}^{21}$ ) which increased to $61.2 \%$ at month 1 and $57.4 \%$ at month 12 postvaccination.

The GMC of $\operatorname{lgM}$ antibodies increased from $24.5 \mathrm{U} / \mathrm{ml}$ prevaccination to $47.5 \mathrm{U} / \mathrm{ml}$ at month $1(p<0.0001)$ and $29.6 \mathrm{U} / \mathrm{ml}(\mathrm{p}=0.8)$ at month 12 postvaccination. Compared to 
median IgM antibody concentrations in the reference group, prevaccination the ratio of the medians was 0.5 , which increased to 1.4 at month 1 and decreased to 0.7 at month 12 postvaccination. At prevaccination date, $26.5 \%$ of patients had global IgM antibody concentrations comparable to the reference group (defined as $\geq 54.0 \mathrm{U} / \mathrm{ml}^{22}$ ) which increased to $55.1 \%$ at month 1 and $28.6 \%$ at month 12 postvaccination.

The GMC of IgA antibodies increased from $13.4 \mathrm{U} / \mathrm{ml}$ prevaccination to $38.4 \mathrm{U} / \mathrm{ml}$ at month $1(p<0.0001)$ and $23.6(p=0.003)$ at month 12 postvaccination. Compared to the reference group, prevaccination the ratio of the medians was 0.6 , which increased to 1.6 at month 1 and 1.1 at month 12 post vaccination. At prevaccination date, $26.5 \%$ of patients had global IgA antibody concentrations comparable to the reference group (defined as $\geq 21.0 \mathrm{U} / \mathrm{ml}^{22}$ ) which increased to $67.3 \%$ at month 1 and $51.1 \%$ at month 12 postvaccination.

\subsection{Serotype specific pneumococcal IgG-ELISA}

Forty-five sera from 15 patients were tested, using a WHO standardized serotype specific pneumococcal ELISA for the 13 serotypes covered by Prevenar 13.

GMCs and GMCRs of serotype specific anti-PCP IgG for each serotype are shown in Figure 3 and Suppl. Tab 4. Comparing the difference between month 1 and prevaccination GMCs, a significant increase was observed for all serotypes except for serotype 3. Comparing the difference between month 12 and prevaccination, GMCs in patients remained significantly elevated for all serotypes except serotype 3. GMC and absolute increase was lowest for serotype 3 and highest for serotype 14 . The highest fold increase was reached for serotype 4 . 


\subsection{Correlation of the various pneumococcal antibody assays}

Correlation was defined as very strong $(r \geq 0.7 / r \leq-0.7)$, strong $(r \geq 0.5 / r \leq-0.5)$, moderate $(r \geq 0.3 / r \leq-0.3)$ or weak $(r<0.3 / r>-0.3)$. For OPA titers and serotype specific anti-PCP $\lg G$, correlations are listed if they were significant for at least one point in time for a specific serotype. The details of the date, strength and number of correlations for the OPA as well as global and serotype specific anti-PCP IgG are listed in Tab. 3.

The OPA titers correlated positively with the global and serotype specific anti-PCP IgG concentrations. Higher global anti-PCP IgG correlated with increased summed serotype specific anti-PCP IgG.

Irrespective of the method used, a strong response at month 1 postvaccination correlated with a strong decrease, meaning a higher difference between month 1 and month 12. For OPA titers and serotype specific anti-PCP IgG this correlation was strong for all serotypes. For global anti-PCP immunoglobulins, it was at least moderate.

\subsection{Impact of clinical parameters}

Higher age correlated moderately with decreased OPA titers, serotype specific antiPCP IgG and global anti-PCP IgA at month 1 postvaccination and strongly with higher anti-PCP IgM at all dates.

Higher albuminuria correlated negatively with OPA titers and global anti-PCP-lgG concentrations.

Creatinine concentration and estimated glomerular filtration rate (eGFR, calculated by CKD-EPI, Suppl. Figure 1) did not correlate significantly with pneumococcal antibodies.

For binary-coded parameters Mann-Whitney $U$ test was performed. 
Patients with vs. without mycophenolate mofetil (MMF) treatment responded to vaccination with decreased OPA titers, global and serotype specific anti-PCP IgG, $\lg \mathrm{G} 2$ and $\lg \mathrm{A}$ at month 1 and 12 postvaccination.

Patients receiving tacrolimus showed increased OPA titers and serotype specific antiPCP IgG. Furthermore, they displayed higher IgA-concentrations 12 months after vaccination.

Patients with an interval between transplantation and vaccination $\geq 12$ months displayed higher OPA titers, as well as higher anti-PCP IgA at month1 post vaccination and higher anti-PCP IgM at all dates compared to those with a shorter interval.

Patients who had received PPSV23 at least 5 years before enrollment had higher OPA concentrations. Patient gender had no significant impact on pneumococcal antibodies.

\section{Discussion}

Altogether PCV13 was demonstrated to be immunogenic in KTR, with significantly increased antibody function and concentrations up to one year postvaccination.

\subsection{Definition of vaccine response criteria}

Recommended endpoints for vaccine non-inferiority were published by the WHO in $2012^{23}$. For primary analysis serotype specific ELISA, with determination of the percentage of subjects with serotype-specific $\lg G \geq 0.35 \mu \mathrm{g} / \mathrm{ml}$ for infants, should be applied. For secondary analysis, the serotype specific OPA titers (GMT) and titer ratios (GMTR) are recommended. Since the serotype specific ELISA method was available for only a subset of our patients, we mainly considered the OPA-values. The threshold of an OPA titer $\geq 1: 8$ has been shown to confer protection in a mouse model ${ }^{24}$. A recent study defining protective OPA titers in children has questioned this general cutoff ${ }^{25}$. In adults, the use of any OPA threshold for defining protection is based on limited data $^{26,27}$, but nevertheless the method is widely accepted as the best functional 
correlate of protection ${ }^{24,25}$. Serological correlates of protection and vaccine response criteria for adults - and immunocompromised patients in particular - remain poorly defined for all methods of vaccine evaluation ${ }^{28,29}$.

\subsection{Results of the opsonophagocytic killing assay}

Dendle et al. have recently reported increased anti-PCP IgG concentrations for all serotypes measured by means of the serotype specific ELISA in 45 adult KTR vaccinated with PCV13 and increased OPA titers for the 4 most immunogenic serotypes ${ }^{12}$. They described the highest proportion of responders, defined as anti-PCP $\operatorname{lgG} \geq 1,0 \mu \mathrm{g} / \mathrm{ml}$ and an $\geq 2$-fold post vaccination, for serotype $19 \mathrm{~A}$ and $18 \mathrm{C}$ and the lowest for serotype 3 and $14^{12}$. However, as in our work, absolute concentrations for serotype 14 were highest. In contrast to our findings, serotype 4 was less immunogenic. Their comparison of KTR (aged 47-63) and the reference group aged 60-64 revealed 15-500 times lower OPA GMTs for the 4 most immunogenic serotypes in KTR 4 weeks post vaccination ${ }^{12}$, while in our population OPA GMTs were 57 times lower at most. The compared populations were not age matched, OPA assays were not performed in the same laboratory and influencing clinical parameters were not evaluated. Since the 4 examined serotypes assessed by the OPA were selected by their ELISA result, the validity of the results is limited.

Kumar et al. compared PCV7 with PPSV23 in 60 adult KTR (30 in each group) and reported response rates defined by an OPA titer $\geq 1: 8$ and a 4 -fold increase in OPA titers for individual serotypes of $37-53 \%{ }^{14} .21 \%$ of patients initially responding to at least 1 serotype, lost their response within 3 years ${ }^{13}$. When applying this definition, the patients of our study displayed similar response rates at month 1 postvaccination. Kumar et al., however, used a flow-cytometry OPA, measuring titers as the reciprocal of the highest serum dilution yielding $50 \%$ of maximum phagocytic uptake ${ }^{14}$. This form 
of OPA is considered to be less biologically relevant than the killing OPA utilized in our study ${ }^{24}$.

So far, no study compared age-matched OPA titers of KTR and healthy adults. Comparing the median GMTR of reference groups and kidney transplant recipients of matched age groups, KTR showed an impaired postvaccination response. The higher prevaccination GMTs for several serotypes in KTR aged 18-49 may have been the result of $35 \%$ of them being prevaccinated with PPSV23 at least 5 years before vaccination with PCV13, in contrast to only $25 \%$ aged $\geq 60$. But when looking at the high prevaccination OPA titers of serotype 7F in kidney transplant recipients aged 1849 for example, only 3 of 10 patients (30\%) showing OPA titers $>200$, contributing to the high GMT of 358 in this group, were prevaccinated with PPSV23 at least 5 years before PCV13. More likely, the high prevaccination titers have been acquired by natural exposure to serotype $7 \mathrm{~F}$ of S. pneumoniae. This is not unusual, for many unimmunized adults display OPA titers $\geq 1: 8$, due to natural exposure to pneumococcal antigens ${ }^{27}$. Although adult KTR responded with significantly increased GMTs to the OPA, they displayed a 5- (age 18-49 years) up to 10-fold (age $\geq 50$ years) decreased initial response to PCV13 when compared with the reference groups. This indicates impairment in antibody function that increases even further with age. A GMCR of 2-3 for serotype specific $\lg G$ has been considered to be the threshold for clinically relevant differences ${ }^{30}$. So the 5-10-fold reduced OPA titers may well be clinically relevant.

\subsection{Results of the global ELISA}

The Vacczyme ${ }^{\odot}$ assay measures the global anti-PCP antibodies against 23 serotypes. The global anti-PCP immunoglobulin concentration resembles the general capability of the immune system to react to polysaccharide-protein conjugate vaccines by using the fold increase and/or the absolute concentrations as criteria for the serological response $^{29}$. The data on normal global anti-PCP-antibody concentrations against 23 
serotypes in healthy adults pre- and postvaccination is limited and there are no validated protective concentrations available ${ }^{31}$. The comparison with the available reference concentrations of vaccine naïve healthy adults ${ }^{21}$, reveals reduced prevaccination concentrations in KTR. Comparable concentrations to healthy adults are reached at month 1 post vaccination and persist until month 12 post vaccination, except for IgM. The global anti-PCP IgG ELISA strongly correlates with summed postvaccination OPA titers and very strongly with summed postvaccination serotype specific ELISA and may be used as an easy and cost-effective tool for the clinical monitoring of the response to pneumococcal vaccination ${ }^{31}$ although it does not provide any information about serotype specific protection. Values below the presented threshold should be re-evaluated with the serotype specific ELISA ${ }^{31}$. A more differentiated view on the immunocompetence may be achieved by the additional assessment of $\lg G 2$, $\lg A$ and $\lg M$. Low $\lg M$ antibody concentrations correlate with higher incidence of pneumonia and bronchiectasis in CVID patients ${ }^{32}$. IgM concentrations have been reported to decrease significantly with age $>60$, while IgG and IgA have been found to increase beyond this age ${ }^{22}$. The results of our study concur on IgM concentrations being strongly affected by patients' age, while not showing any significant correlation with $\lg G, \lg G 2$ and $\lg A$ at any time. Further investigation on the role of immunoglobulin subclasses in pneumococcal immunity is needed.

\subsection{Results of the serotype specific ELISA}

Tobudic et al. previously analyzed 33 KTR after a prime-boost vaccination with PCV7 and PPSV23 by serotype specific ELISA ${ }^{10}$. They reported a response to at least one serotype in $77.1 \%$ of patients, defined by a 2 -fold increase in antibody concentration and an absolute postvaccination value $\geq 1 \mu \mathrm{g} / \mathrm{ml}$ in the serotype specific WHOreference ELISA ${ }^{10}$. No OPA was used in this study. Their result of serotype 14 inducing the highest antibody concentrations was reproduced by both OPA and ELISA in our 
study. Our finding that serotype 3 elicits a weak humoral response in both OPA and ELISA corresponds with the results of other studies ${ }^{25,33}$.

\subsection{Correlation between OPA and ELISA}

OPA and serotype specific ELISA have been shown to correlate well in children, but poorly in adults ${ }^{24,28}$. In KTR and immunocompromised individuals, studies indicate contradictory results. Tarragó et al. found no correlation of serotype specific flow cytometric OPA and IgG ELISA in KTR immunized with PPSV23 for 3 serotypes ${ }^{34}$. Karlsson et al. presented poor correlation of serotype specific killing OPA and IgG ELISA for 4 serotypes in vaccinated patients with multiple myeloma and Waldenstrom's macroglobulinemia ${ }^{35}$. In contrast, Kumar et al. reported moderate but significant correlations for 6 of 7 serotypes using flow cytometric OPA ${ }^{14}$. In our study, correlations were strong and significant for only a minority of serotypes but positive for all serotypes except for 6B. Correlation coefficients improved after vaccination. While most patients experienced a significant increase of both OPA titers and IgG-concentrations, the increase in OPA titers was often predominant. There were even some patients and serotypes in which we observed extensive postvaccination increase of OPA titers while $\lg$ concentrations remained similar to prevaccination levels (Suppl. Fig 2). The explicit reasons for this OPA - ELISA discordance in adults are unknown. High OPA titers in serum with low anti-PCP IgG could be explained by an increased antibody quality leading to enhanced biological function. However, other immunoglobulin subclasses than IgG may substantially contribute to the OPA tite ${ }^{27}$, since we observed increased correlation coefficients when correlating OPA titers with summed global immunoglobulins compared to global IgG alone. Possible additional influencing factors are non-PCP-specific OPA killing activity and antibodies against pneumococcal protein antigens $^{36}$ - which are currently being evaluated for their potential inclusion in 
pneumococcal vaccines. Both have been reported to be present in unvaccinated individuals ${ }^{37}$ and may consequently be less relevant for a postvaccination response.

\subsection{Impact of immunosuppression}

Prednisone, cyclosporine, tacrolimus and MMF are the most commonly used immunosuppressive agents in kidney transplant recipients ${ }^{38,39}$. Our findings correspond well with the dominant impact of MMF on humoral immune response, compared to other immunosuppressive drugs, that has already been described ${ }^{39,40}$.

Our study has some limitations. First the sample size was rather small, which may have led to missing smaller differences in vaccine response and influencing factors. Secondly, we could perform the serotype specific ELISA for only a subset of patients. By measuring samples with three methods though, we could analyze their correlation.

\subsection{Conclusions}

This study reveals moderate but significant immunogenicity of the 13-valent pneumococcal conjugate vaccine in kidney transplant recipients (KTR). Although consensual vaccine response definitions are lacking in the literature, our results indicate increased postvaccination humoral responses with increased antibody function for at least one year postvaccination. Compared to healthy adults, KTR display 5-10-fold decreased opsonic antibody function. After vaccination initially low concentrations of global anti-pneumococcal capsular polysaccharide lgG, IgG2, IgM and $\lg A$ are increased to levels similar to those seen in healthy unvaccinated adults. 


\section{Methods}

\subsection{Study design and population}

49 clinically stable adult kidney transplant recipients were included in this single center trial. After the blood withdrawal at month 1, two patients dropped out, one deceased and one was no longer available because he moved away. Inclusion criteria were: absence of clinical infection, stable kidney function defined as $<15 \%$ change in serum creatinine within 1 month prior to vaccination, an interval of $\geq 4$ months to kidney transplantation and absence of pregnancy. Baseline characteristics of all patients are listed in Tab. 1 and Suppl. Fig. 1.

\subsection{Vaccine}

13-valent PCV (PCV13/Prevenar $13^{\circledR}$, Pfizer, New York, NY, USA) contains polysaccharides of pneumococcal serotypes $1,3,4,5,6 \mathrm{~A}, 6 \mathrm{~B}, 7 \mathrm{~F}, 9 \mathrm{~V}, 14,18 \mathrm{C}, 19 \mathrm{~F}$, $19 \mathrm{~A}$ and $23 \mathrm{~F}$, individually conjugated to a nontoxic mutant form of diphtheria toxin cross-reactive material 197 (CRM197). Each $0.5 \mathrm{ml}$ dose contains $2.2 \mu \mathrm{g}$ of each serotype, except type 6B, which is included at $4.4 \mu \mathrm{g}$. Each dose is formulated in 5.0 $\mathrm{mM}$ succinate and $0.85 \%$ sodium chloride at $\mathrm{pH} 5.8$ with $0.125 \mathrm{mg}$ aluminum as aluminum phosphate and $0.02 \%$ polysorbate 80 . The vaccine is supplied in singledose syringes without preservatives and stored at $2-8^{\circ} \mathrm{C}$. Vaccines were administered by intramuscular injection into the deltoid.

\subsection{Immunogenicity assessment}

Blood was drawn immediately prevaccination (T0) and at month 1 (T1) and month 12 (T2) postvaccination and kept frozen $\left(-80^{\circ} \mathrm{C}\right)$ in $1 \mathrm{ml}$ aliquots. Primary endpoint was the determination of serotype specific functional antibacterial opsonophagocytic 
response, by means of a validated opsonophagocytic killing assay (OPA). The OPA is generally accepted as the best functional correlate of pneumococcal immunity ${ }^{41}$. The details of the OPA-protocol have been described elsewhere ${ }^{41}$. The interpolated reciprocal serum dilution that resulted in complement-mediated killing of $50 \%$ of the assay bacteria was defined as the OPA titer. The lower limit of quantitation (LLOQ) was $>1: 8$ and was determined during assay validation for each serotype. Values below the LLOQ were defined as half of the LLOQ. Serum samples of all 49 patients were shipped to the Pfizer Vaccines Research Laboratory (New York, NY, U.S.A.) for the performance of the OPA.

In addition, for all 49 kidney transplant recipients receiving Prevnar 13, the total amount of anti-pneumococcal antibodies against 23 serotypes was measured by enzyme linked immunosorbent assay (here called "global ELISA") for IgG, IgG2, IgM and IgA (Vacczyme ${ }^{\odot}$, The Binding Site, Schwetzingen, Germany). The global pneumococcal ELISA provides an orientation on the humoral capability to respond to vaccines ${ }^{42}$.

Based on the global IgG ELISA results, samples of 5 patients with high, 5 with intermediate and 5 with low antibody concentrations postvaccination were selected and shipped to the WHO Pneumococcal Serology Reference Laboratory (London, UK). Serotype-specific IgG levels were measured by means of the World Health Organization reference ELISA after adsorption with cell wall and 22F polysaccharide ${ }^{43}$. The LLOQ of the ELISA was $0.150 \mu \mathrm{g} / \mathrm{ml}$ for all serotypes. Results lower than the LLOQ were declared as half of the LLOQ $(0.075 \mu \mathrm{g} / \mathrm{ml})$.

\subsection{Immunogenicity analyses}

A positive response to one serotype was defined as OPA titer $\geq 1: 8 .{ }^{44}$ According to a paper by Orange et al., a patient was defined as responder if the response criteria were met for at least $9 / 13$ serotypes $(70 \%)$ at month 1 postvaccination. ${ }^{18}$ For the evaluation 
of the geometric mean titer ratios post vs. prior to vaccination, a four-fold increase, as a rather strict criterion, was considered as a positive response ${ }^{14,45}$.

For comparison, two US studies on PCV13-vaccination ${ }^{19,20}$ with vaccine-naïve healthy adults of different age groups were chosen. GMTs had been analyzed with the same method, at the same laboratory as our patients. The first study assessed the immunogenicity and safety of PCV13 in adults $18-49$ and $60-64$ years of age ${ }^{19}$. The healthy adults were analyzed prevaccination and at month 1 and 12 postvaccination, as in our current study. The second study was designed as the first but included adults 50-59 years of age and reported GMTs only for month 1 postvaccination ${ }^{20}$. Altogether, three age groups of a healthy reference population and kidney transplant recipients were compared. Since our study population in the group aged 60-64 comprised only 5 patients, all patients $\geq 60(n=16)$ were compared with the healthy adults aged 60-64. About $30 \%$ of our KTR were prevaccinated with PPSV23 at least 5 years before vaccination with PCV13.

The references for global IgG and IgG2, IgM and IgA ELISA concentrations are from two studies of healthy, vaccine naïve blood donors ${ }^{21,22}$.

\subsection{Statistical analysis}

Statistical analysis was performed with Graph Pad Prism 6.0 $0^{\odot}$ for Windows (GraphPad Prism Software, La Jolla, CA, U.S.A.). Characteristics of responders, non-responders and of healthy adults and kidney transplant recipients were compared by MannWhitney test. Wilcoxon signed rank test for paired samples was performed, comparing the difference of prevaccination with month 1 or month 12 postvaccination, respectively. This was done for the results of the OPA, the global ELISA and the serotype specific ELISA, respectively. $P$ values of $<0.05$ were considered as 
significant. An exploratory analysis of the impact of clinical parameters was carried out using Spearman correlation analysis or Mann-Whitney test as appropriate.

\section{Tables}

\begin{tabular}{|c|c|c|}
\hline Clinical parameters and vaccine response & $\begin{array}{l}\text { Responder } \\
(\mathrm{n}=26)\end{array}$ & $\begin{array}{l}\text { Non-Responder } \\
(\mathrm{n}=23)\end{array}$ \\
\hline Male & 17 & 14 \\
\hline Female & 9 & 9 \\
\hline Age (years; median, range) & $53(24-72)$ & $59(21-73)$ \\
\hline Creatinine (mg/dl; median, range) & $1.7(1.1-3.3)$ & $1.7(0.9-4.9)$ \\
\hline eGFR by CKD-EPI (ml/min; median, range) & $37(20-89)$ & $43(11-66)$ \\
\hline Albuminuria (mg/g creatinine; median, range) & $77(0.3-655)$ & $140(0.3-3104)$ \\
\hline $\begin{array}{l}\text { Interval transplantation - vaccination } \\
\text { (months; median, range) }\end{array}$ & $52(4-260)$ & $41(8-413)$ \\
\hline Immunosuppressive drugs (median number, range) & $3(2-3)$ & $3(1-3)$ \\
\hline Global IgG ELISA at month $1 \geq 43.8 \mathrm{mg} / \mathrm{l}$ (number of patients, \%) & $25(96) * \star \star$ & $9(39) * \star \star$ \\
\hline Global IgG2 ELISA at month $1 \geq 20.5 \mathrm{mg} / \mathrm{l}$ (number of patients, \%) & $23(88) * \star \star$ & $7(30) * \star \star$ \\
\hline Global IgM ELISA at month $1 \geq 54.0 \mathrm{U} / \mathrm{ml}$ (number of patients, \%) & $18(69)$ * & $9(39) *$ \\
\hline Global IgA ELISA at month $1 \geq 21.0 \mathrm{U} / \mathrm{ml}$ (number of patients, \%) & $24(92) * * *$ & $9(39) * * \star$ \\
\hline $\begin{array}{l}\text { Previous vaccination ( }>5 \text { years ago with PPSV23; number of } \\
\text { patients, \%) }\end{array}$ & $7(27)$ & $8(35)$ \\
\hline Prednison (number of patients, \%) & $25(96)$ & $21(91)$ \\
\hline Tacrolimus (number of patients, \%) & $19(73)$ & $14(61)$ \\
\hline Cyclosporine (number of patients, \%) & $6(23)$ & $4(17)$ \\
\hline MMF (number of patients, \%) & $16(62)$ & $19(83)$ \\
\hline Sirolimus/Everolimus (number of patients, \%) & $2(8)$ & $2(9)$ \\
\hline Eculizumab (number of patients, \%) & $2(8)$ & $0(0)$ \\
\hline $\begin{array}{l}\text { Acute kidney failure within follow-up of } 12 \text { months number of } \\
\text { patients, \%) }\end{array}$ & $3(12)$ & $2(9)$ \\
\hline Rejection within follow-up of 12 months (number of patients, \%) & $2(8)$ & $2(9)$ \\
\hline $\begin{array}{l}\text { Humoral rejection within follow-up of } 12 \text { months (number of } \\
\text { patients, \%) }\end{array}$ & $1(4)$ & $1(4)$ \\
\hline $\begin{array}{l}\text { Cellular and borderline rejection within follow-up of } 12 \text { months } \\
\text { (number of patients, \%) }\end{array}$ & $1(4)$ & $1(4)$ \\
\hline
\end{tabular}

Tab. 1: Study population comparing responders and non-responders in respect of clinical parameters a Responders reached opsonophagocytic killing assay (OPA) titers $\geq 1: 8$ for at least $9 / 13(=70 \%)$ serotypes Clinical parameters (creatinine, eGFR, albuminuria, immunosuppressive drugs, MMF, sirolimus/everolimus data collected at prevaccination date). Severe events like episodes of acute kidney failure or rejection are lis 
of 12 months. Differences between the groups were analyzed by Mann-Whitney test. Significant differences groups are labeled as follows:

${ }^{*} p<0.05,{ }^{* *} p<0.005,{ }^{* * *} p<0.0001$.

a Percentage of patients reaching anti-PCP immunoglobulin concentrations comparable to healthy referenc eGFR, estimated glomerular filtration rate (estimated by CKD-EPI-formula); IgG; immunoglobulin immunosorbent assay; PPSV23, 23-valent pneumococcal polysaccharide vaccine; MMF, mycophenolate $m$ 


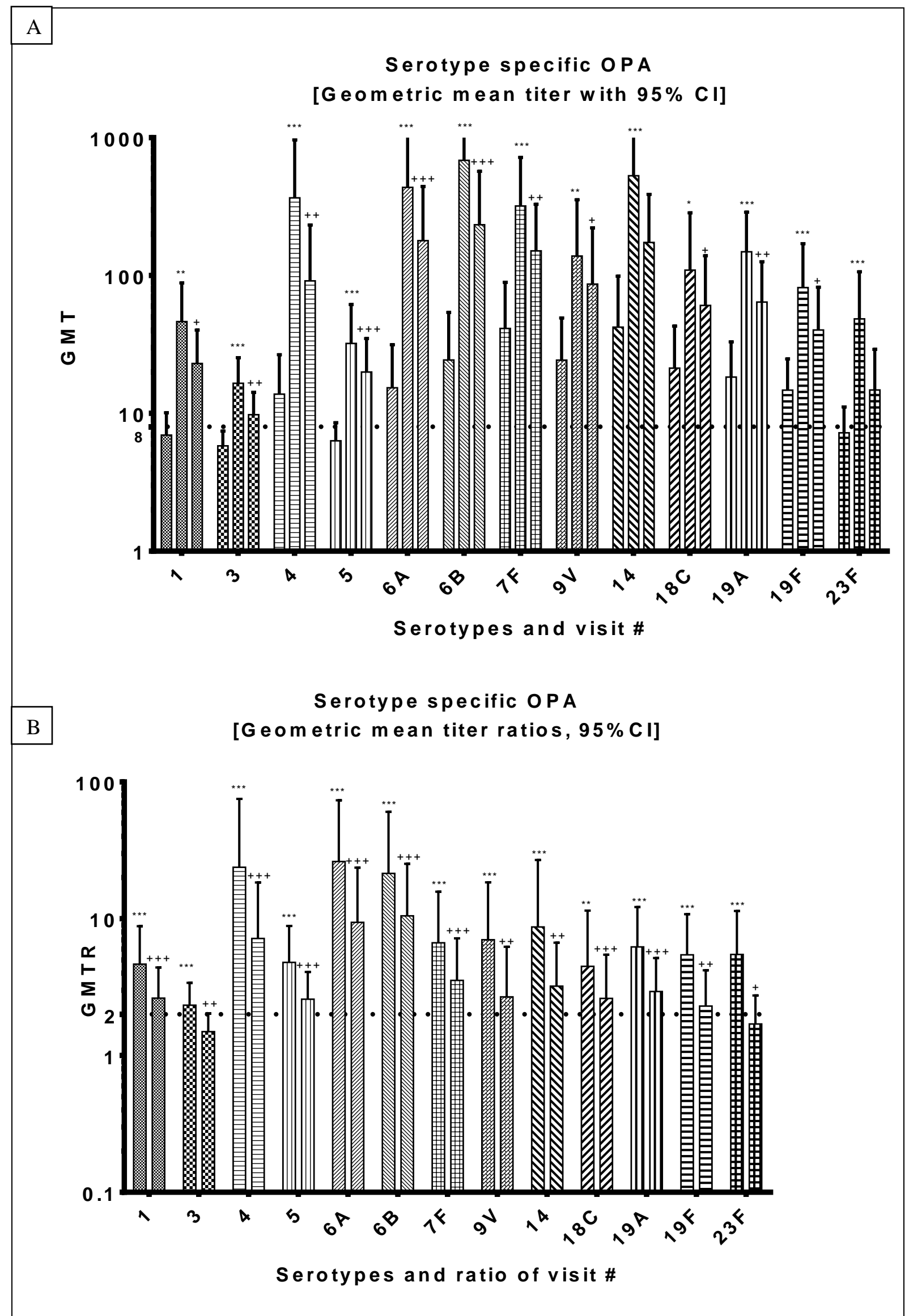

Figure 1: Significant increase of serotype specific geometric mean opsonophagocytic antibody titers/ratios against 13 serotypes in 145 serum samples of 49 kidney transplant recipients (49 prevaccination, 49 month 1 and 47 month 12 postvaccination). Geometric 
mean titers (GMTs) with 95\% confidence interval (A) are shown at prevaccination date (T0), at month 1 (T1) and month 12 postvaccination (T2). The dotted line at an OPA titer of 8 represents the cut-off for a positive response ${ }^{18}$. Geometric mean titer ratios (GMTR) with 95\% confidence interval (month 1 or month 12 postvaccination divided by prevaccination level) are shown in (B). The dotted line at a GMTR of 2 represents the cut-off for a positive response ${ }^{18}$. Using Wilcoxon signed rank matched pairs test, data at months 1 and 12 were compared with prevaccination values. Comparison of prevaccination values with month 1: * $p<0.05,{ }^{* *} p<0.005,{ }^{* * *} p<0.0001$; comparison with month $12:+p<0.05++p<0.005,+++$ $\mathrm{p}<0.0001$.

OPA, opsonophagocytic killing assay

\begin{tabular}{|c|c|c|c|}
\hline \multicolumn{4}{|c|}{$\begin{array}{l}\text { Geometric mean titer ratios (GMTRs) }{ }^{a} \text { in healthy adult reference } \\
\text { populations }{ }^{19,20} \text { and kidney transplant recipients, divided by age } \\
\text { group }\end{array}$} \\
\hline \multicolumn{4}{|c|}{\begin{tabular}{l|l|l|l} 
& Prevaccination & Month 1 & Month 12 \\
\end{tabular}} \\
\hline \multicolumn{4}{|c|}{\begin{tabular}{l|l} 
Month 1 & Month 12 \\
4.8 & 2.6
\end{tabular}} \\
\hline Age $50-59$ & & 10.0 & \\
\hline Age $\geq 60$ & 1.1 & 10.1 & 5.2 \\
\hline
\end{tabular}

Tab. 2: Comparison of geometric mean titer ratios (GMTRs) of healthy adults (HA) of reference populations from 2 US studies and kidney transplant recipients $(\mathrm{HA} / \mathrm{KTR})$. Patients were divided by age (18-49, 5059 and $\geq 60$ ). The medians of GMTRs prevaccination and at month 1 and 12 postvaccination are depicted.

a Ratios are geometric mean titer ratios, defined by opsonophagocytic killing assay (OPA). They were calculated by back-transforming the mean difference between age groups on the logarithmic scale. 


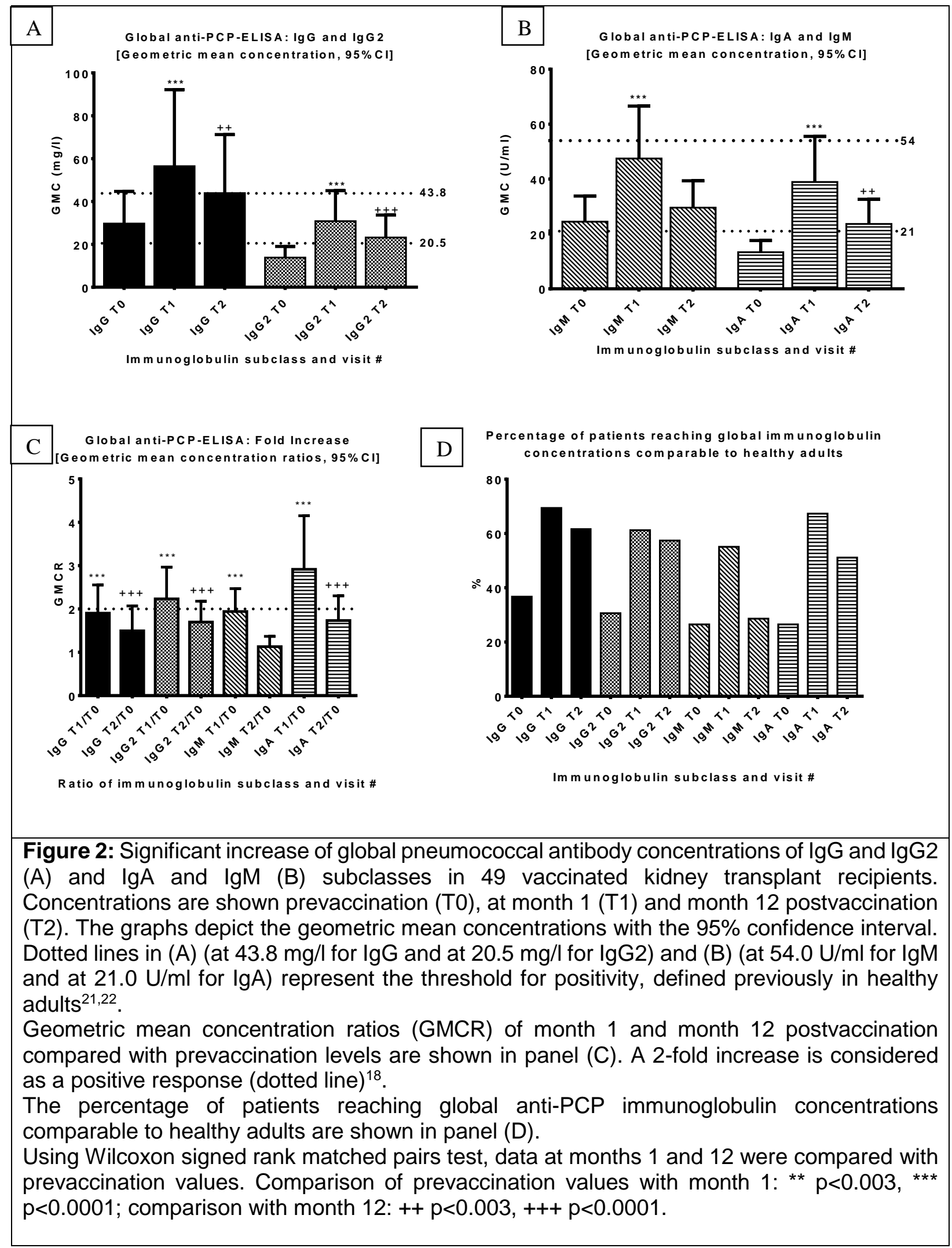




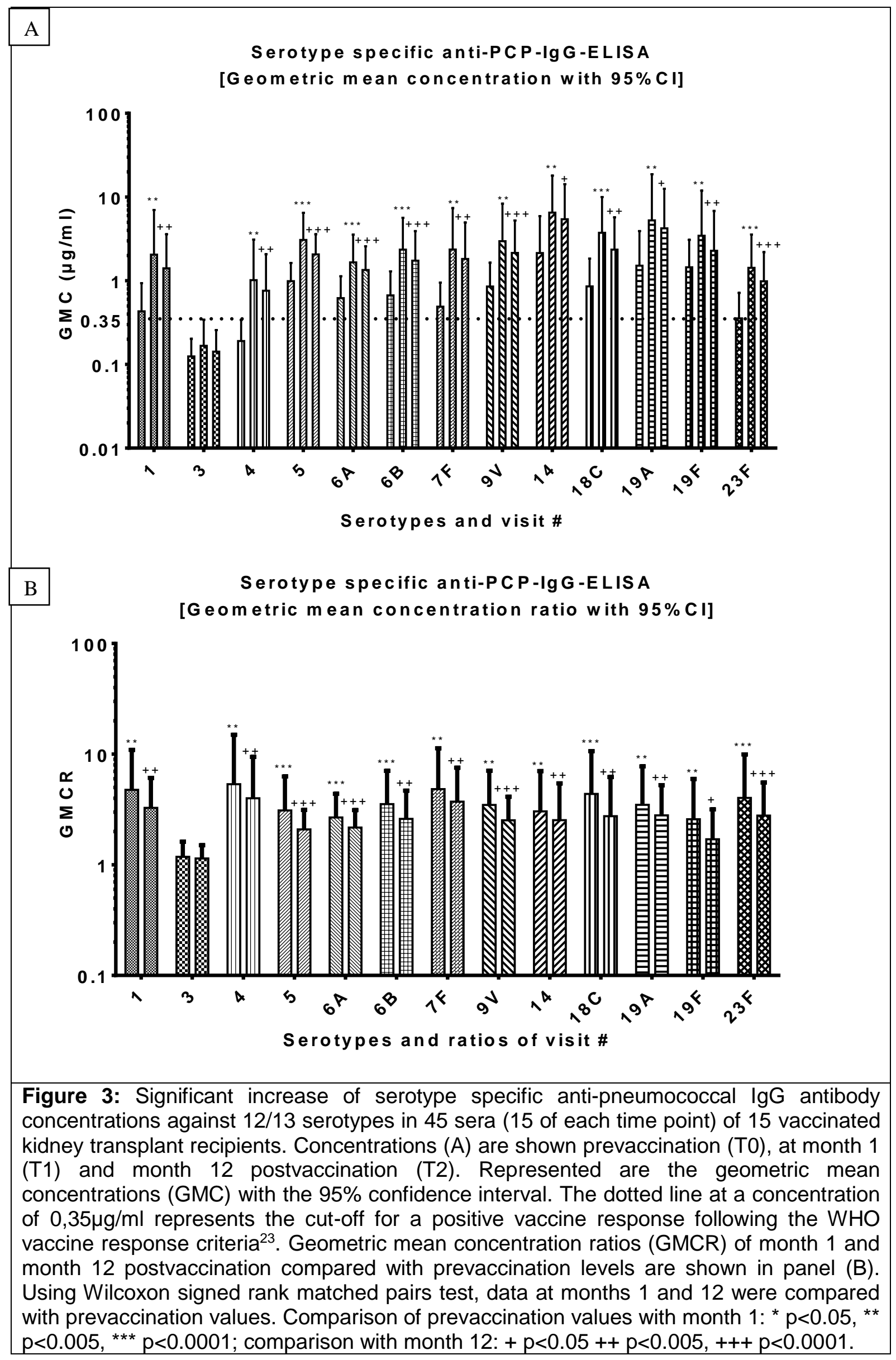




\begin{tabular}{|c|c|c|c|c|c|c|c|c|c|}
\hline & \multicolumn{3}{|c|}{ Serotype-specific OPA } & \multicolumn{3}{|c|}{ Global IgG ELISA } & \multicolumn{3}{|c|}{ Serotype-specific IgG ELISA } \\
\hline & Prevaccination & \begin{tabular}{|l|} 
Month \\
1 \\
\end{tabular} & \begin{tabular}{|l|} 
Month \\
12 \\
\end{tabular} & Prevaccination & $\begin{array}{l}\text { Month } \\
1\end{array}$ & $\begin{array}{l}\text { Month } \\
12\end{array}$ & Prevaccination & \begin{tabular}{|l|} 
Month \\
1
\end{tabular} & \begin{tabular}{|l} 
Month \\
12 \\
\end{tabular} \\
\hline $\begin{array}{l}\text { Global IgG } \\
\text { ELISA }\end{array}$ & $t^{a}$ & $++^{\mathrm{a}}$ & $+^{\mathrm{a}}$ & & & & $++^{a}$ & $+++^{\mathrm{a}}$ & $+++^{a}$ \\
\hline $\begin{array}{ll}\text { Serotype- } & \\
\text { specific } \\
\text { ELISA }\end{array}$ & $\begin{array}{l}++++^{\mathrm{b}} \\
(5 / 13)\end{array}$ & $\begin{array}{l}++^{\mathrm{b}} \\
(4 / 13)\end{array}$ & $\begin{array}{l}++++^{\mathrm{b}} \\
(2 / 13)\end{array}$ & $++^{\mathrm{a}}$ & $+++^{a}$ & $+++^{a}$ & & & \\
\hline Albuminuria & $\begin{array}{l}- \\
(2 / 13)\end{array}$ & $\begin{array}{l}- \\
(3 / 13)\end{array}$ & $\begin{array}{l}-- \\
(6 / 13)\end{array}$ & & -- & --- & & & \\
\hline $\begin{array}{l}\text { Mycophenolate } \\
\text { mofetil cd }\end{array}$ & $(1 / 13)$ & $(9 / 13)$ & $(5 / 13)$ & & - & - & $\begin{array}{l}- \\
(1 / 13)\end{array}$ & $\begin{array}{l}- \\
(13 / 13)\end{array}$ & $\begin{array}{l}- \\
(8 / 13)\end{array}$ \\
\hline Tacrolimus $^{c}$ & $\begin{array}{l}+ \\
(2 / 13)\end{array}$ & $\begin{array}{l}+ \\
(1 / 13)\end{array}$ & & & & & $\begin{array}{l}+ \\
(3 / 13)\end{array}$ & $\begin{array}{l}+ \\
(7 / 13)\end{array}$ & $\begin{array}{l}+ \\
(4 / 13)\end{array}$ \\
\hline $\begin{array}{l}\text { Interval } \\
\text { between } \\
\text { transplantation } \\
\text { and } \\
\text { vaccination } \\
>12 \text { months }\end{array}$ & & $\begin{array}{l}+ \\
(7 / 13)\end{array}$ & $\begin{array}{l}+ \\
(7 / 13)\end{array}$ & & & & & & \\
\hline $\begin{array}{l}\text { Previous } \\
\text { pneumococcal } \\
\text { vaccination >5 } \\
\text { years }\end{array}$ & & $\begin{array}{l}+ \\
(2 / 13)\end{array}$ & $\begin{array}{l}+ \\
(1 / 13)\end{array}$ & & & & & & \\
\hline Age & $\begin{array}{l}-- \\
(4 / 13)\end{array}$ & $\begin{array}{l}-- \\
(6 / 13)\end{array}$ & $\begin{array}{l}-- \\
(9 / 13)\end{array}$ & & & & $\begin{array}{l}-- \\
(1 / 13)\end{array}$ & $\begin{array}{l}-- \\
(1 / 13)\end{array}$ & $\begin{array}{l}-- \\
(1 / 13)\end{array}$ \\
\hline \multicolumn{10}{|c|}{$\begin{array}{l}\text { Tab. 3: Impact of various parameters on humoral immunity (opsonophagocytic killing assay (OPA), global and serotype- } \\
\text { specific ELISA, measured prevaccination and at month } 1 \text { and } 12 \text { postvaccination). Data were either analyzed by Spearman } \\
\text { correlation analysis or Mann-Whitney } U \text { test as appropriate. Correlation data: }+-, r \geq 0.3 / r \leq-0.3 ;++/--, r \geq 0.5 / r \leq-0.5 ;+++/--, \\
r \geq 0.7 / r \leq-0.7 \text {. For parameters analysed with Mann-Whitney } U \text { test, the difference of the medians is labeled as }+ \text { (positive } \\
\text { difference for those featuring the character) or }- \text { (negative difference for those featuring the character). } \\
\text { ( } x / 13 \text { ), number of } x \text { out of the } 13 \text { measured serotypes showing significant results }(p<0.05) \text {. } \\
\text { From varying correlation coefficients, the maximum is displayed. All displayed correlations were at least moderate }(r \geq 0.3 / r \leq- \\
0.3) \text {. Two parameters/data sets were correlated always at the same timepoint (Spearman correlation analysis). } \\
\text { Correlations that were significant for a single method and one serotype only are not displayed. } \\
\text { a. OPA titers of } 13 \text { serotypes and serotype specific IgG concentrations were summed up for individual patients at } \\
\text { each timepoint and then correlated with global IgG ELISA concentrations } \\
\text { b. OPA titers and serotype specific IgG concentrations were correlated by serotype and timepoint } \\
\text { c. Mann-Whitney test of immunosuppression by substance (yes or no), regardless of dose }\end{array}$} \\
\hline
\end{tabular}

\section{Disclosure}

https://www.elsevier.com/_data/promis_misc/KI_Author_disclosure_form.pdf 


\section{Acknowledgements}

We are grateful to Barbara Nilewski, Tanja Hinkeldein and Martina Praast for their excellent technical assistance.

\section{References}

1. Rubin RH, Hirsch HH. Transplant infectious disease: a moving target. Transplant infectious disease : an official journal of the Transplantation Society. 2008;10(1):1-2.

2. Bonatti $\mathrm{H}$, Pruett TL, Brandacher G, et al. Pneumonia in solid organ recipients: spectrum of pathogens in 217 episodes. Transplantation proceedings. 2009;41(1):371-374.

3. Musher DM, Abers MS, Bartlett JG. Evolving Understanding of the Causes of Pneumonia in Adults, With Special Attention to the Role of Pneumococcus. Clinical infectious diseases : an official publication of the Infectious Diseases Society of America. 2017;65(10):1736-1744.

4. Shigayeva A, Rudnick W, Green K, et al. Invasive Pneumococcal Disease Among Immunocompromised Persons: Implications for Vaccination Programs. Clinical infectious diseases : an official publication of the Infectious Diseases Society of America. 2016;62(2):139-147.

5. Arason VA, Sigurdsson JA, Erlendsdottir H, Gudmundsson S, Kristinsson KG. The role of antimicrobial use in the epidemiology of resistant pneumococci: A 10-year follow up. Microbial drug resistance (Larchmont, NY). 2006;12(3):169-176.

6. Pneumococcal Disease | Global Pneumococcal Disease and Vaccine | CDC. https://www.cdc.gov/pneumococcal/global.html. Accessed 12.12.2017.

7. Miller E, Andrews NJ, Waight PA, Slack MPE, George RC. Herd immunity and serotype replacement 4 years after seven-valent pneumococcal conjugate vaccination in England and Wales: An observational cohort study. The Lancet Infectious Diseases. 2011;11(10):760-768.

8. RKI - Pneumoweb-Sentinel - PneumoWeb. https://www.rki.de/DE/Content/Infekt/Sentinel/Pneumoweb/Monatsstatistik.html;jsessionid=9 654F6DDF44D88B4FFC26336AD8DE23B.2_cid372\#doc2378878bodyText3. Accessed 01.11 .2016$.

9. RKI - Impfungen A - Z - STIKO: Wissenschaftliche Begründung für die Aktualisierung der Empfehlungen zur Indikationsimpfung gegen Pneumokokken für Risikogruppen. https://www.rki.de/DE/Content/Infekt/EpidBull/Archiv/2016/37/Art_01.html;jsessionid=A029 B44BFBED619301FD5C6EA682BACC.2 cid372?nn=2375548. Accessed 12.12.2017.

10. Tobudic S, Plunger V, Sunder-Plassmann G, Riegersperger M, Burgmann H. Randomized, single blind, controlled trial to evaluate the prime- boost strategy for pneumococcal vaccination in renal transplant recipients. PloS one. 2012;7(9):e46133.

11. Lindemann M, Heinemann FM, Heinold A, Zaslavskaya M, Horn PA, Witzke O. Pneumococcal antibodies in kidney transplant recipients are predictive of patient survival. Scand J Immunol. 2013;78(6):554-556.

12. Dendle C, Stuart RL, Polkinghorne KR, et al. Seroresponses and safety of 13 -valent pneumococcal conjugate vaccination in kidney transplant recipients. Transpl Infect Dis. 2018;20(2):e12866.

13. Kumar D, Welsh B, Siegal D, Chen MH, Humar A. Immunogenicity of pneumococcal vaccine in renal transplant recipients--three year follow-up of a randomized trial. American journal of transplantation : official journal of the American Society of Transplantation and the American Society of Transplant Surgeons. 2007;7(3):633-638.

14. Kumar D, Rotstein C, Miyata G, Arlen D, Humar A. Randomized, double-blind, controlled trial of pneumococcal vaccination in renal transplant recipients. The Journal of infectious diseases. 2003;187(10):1639-1645. 
15. Lindemann M, Heinemann FM, Horn PA, Witzke O. Immunity to pneumococcal antigens in kidney transplant recipients. Transplantation. 2010;90(12):1463-1467.

16. Lindemann M, Heinemann FM, Horn PA, Witzke O. Long-term response to vaccination against pneumococcal antigens in kidney transplant recipients. Transplantation.

2012;94(1):50-56.

17. Lindemann M, Heinemann FM, Horn PA, Witzke O. Vaccination against Streptococcus pneumoniae does not induce antibodies against HLA or MICA in clinically stable kidney transplant recipients. Human immunology. 2013;74(10):1267-1270.

18. Orange JS, Ballow M, Stiehm ER, et al. Use and interpretation of diagnostic vaccination in primary immunodeficiency: a working group report of the Basic and Clinical Immunology Interest Section of the American Academy of Allergy, Asthma \& Immunology. The Journal of allergy and clinical immunology. 2012;130(3 Suppl):S1-24.

19. Bryant KA, Frenck R, Gurtman A, et al. Immunogenicity and safety of a 13-valent pneumococcal conjugate vaccine in adults 18-49 years of age, naive to 23-valent pneumococcal polysaccharide vaccine. Vaccine. 2015;33(43):5854-5860.

20. Jackson LA, Gurtman A, van Cleeff M, et al. Immunogenicity and safety of a 13-valent pneumococcal conjugate vaccine compared to a 23-valent pneumococcal polysaccharide vaccine in pneumococcal vaccine-naive adults. Vaccine. 2013;31(35):3577-3584.

21. Schauer U, Stemberg F, Rieger CHL, et al. Levels of Antibodies Specific to Tetanus Toxoid, Haemophilus influenzae Type b, and Pneumococcal Capsular Polysaccharide in Healthy Children and Adults. Clinical and Vaccine Immunology. 2003;10(2):202-207.

22. Parker AR, Allen S, Harding S. Concentration of anti-pneumococcal capsular polysaccharide $\operatorname{IgM}, \operatorname{IgG}$ and $\operatorname{IgA}$ specific antibodies in adult blood donors. Practical laboratory medicine. 2016;5:1-5.

23. Pneumococcal Vaccine Position Paper. 2012; www.who.int/entity/wer/2012/wer8714.pdf?ua=1. Accessed 10.08.2016.

24. Romero-Steiner S, Frasch CE, Carlone G, Fleck RA, Goldblatt D, Nahm MH. Use of opsonophagocytosis for serological evaluation of pneumococcal vaccines. Clinical and vaccine immunology : CVI. 2006;13(2):165-169.

25. Andrews NJ, Waight PA, Burbidge P, et al. Serotype-specific effectiveness and correlates of protection for the 13-valent pneumococcal conjugate vaccine: A postlicensure indirect cohort study. The Lancet Infectious Diseases. 2014;14(9):839-846.

26. Westerink MAJ, Schroeder HW, Nahm MH. Immune Responses to pneumococcal vaccines in children and adults: Rationale for age-specific vaccination. Aging and disease. 2012;3(1):5167.

27. Song JY, Moseley MA, Burton RL, Nahm MH. Pneumococcal vaccine and opsonic pneumococcal antibody. Journal of infection and chemotherapy : official journal of the Japan Society of Chemotherapy. 2013;19(3):412-425.

28. Vandecasteele SJ, Ombelet S, Blumental S, Peetermans WE. The ABC of pneumococcal infections and vaccination in patients with chronic kidney disease. Clinical kidney journal. 2015;8(3):318-324.

29. Daly TM, Hill HR. Use and clinical interpretation of pneumococcal antibody measurements in the evaluation of humoral immune function. Clinical and vaccine immunology : CVI. 2015;22(2):148-152.

30. Schwarz TF, Flamaing J, Rümke HC, et al. A randomized, double-blind trial to evaluate immunogenicity and safety of 13 -valent pneumococcal conjugate vaccine given concomitantly with trivalent influenza vaccine in adults aged $\geq 65$ years. Vaccine. 2011;29(32):5195-5202.

31. Rose MA, Buess J, Ventur Y, et al. Reference ranges and cutoff levels of pneumococcal antibody global serum assays (IgG and IgG2) and specific antibodies in healthy children and adults. Medical microbiology and immunology. 2013;202(4):285-294.

32. Cavaliere FM, Milito C, Martini H, et al. Quantification of IgM and IgA anti-pneumococcal capsular polysaccharides by a new ELISA assay: A valuable diagnostic and prognostic tool for common variable immunodeficiency. Journal of clinical immunology. 2013;33(4):838-846.

33. Prymula R, Peeters P, Chrobok V, et al. Pneumococcal capsular polysaccharides conjugated to protein $\mathrm{D}$ for prevention of acute otitis media caused by both Streptococcus pneumoniae and 
non-typable Haemophilus influenzae: A randomised double-blind efficacy study. Lancet (London, England). 2006;367(9512):740-748.

34. Tarragó D, Aguilar L, Jansen WTM, et al. Dependence of correlations between antibody titres and opsonophagocytosis on pneumococcal serotype and patient morbidity in pre- and postpneumococcal vaccination states. Clinical microbiology and infection : the official publication of the European Society of Clinical Microbiology and Infectious Diseases. 2007;13(4):369376.

35. Karlsson J, Roalfe L, Hogevik H, et al. Poor Correlation between Pneumococcal IgG and IgM Titers and Opsonophagocytic Activity in Vaccinated Patients with Multiple Myeloma and Waldenstrom's Macroglobulinemia. Clinical and vaccine immunology : CVI. 2016;23(4):379_ 385.

36. Pichichero ME, Khan MN, Xu Q. Next generation protein based Streptococcus pneumoniae vaccines. Human vaccines \& immunotherapeutics. 2016;12(1):194-205.

37. Hu BT, Yu X, Jones TR, et al. Approach to validating an opsonophagocytic assay for Streptococcus pneumoniae. Clinical and diagnostic laboratory immunology. 2005;12(2):287295.

38. Türk TR, Witzke O, Zeier M. KDIGO-Leitlinien zur Betreuung von Nierentransplantatempfängern. Der Nephrologe. 2010;5(2):94-107.

39. Legris T, Picard C, Moal V, et al. Humoral immunity after kidney transplantation: impact of two randomized immunosuppressive protocols. Annals of transplantation. 2013;18:622-634.

40. Meerveld-Eggink A, van der Velden AMT, Ossenkoppele GJ, van de Loosdrecht AA, Biesma DH, Rijkers GT. Antibody response to polysaccharide conjugate vaccines after nonmyeloablative allogeneic stem cell transplantation. Biology of blood and marrow transplantation : journal of the American Society for Blood and Marrow Transplantation. 2009;15(12):1523-1530.

41. Cooper D, Yu X, Sidhu M, Nahm MH, Fernsten P, Jansen KU. The 13-valent pneumococcal conjugate vaccine (PCV13) elicits cross-functional opsonophagocytic killing responses in humans to Streptococcus pneumoniae serotypes 6C and 7A. Vaccine. 2011;29(41):7207-7211.

42. VaccZyme ${ }^{\mathrm{TM}}$ Enzym-Immunoassay zur VaccZyme ${ }^{\mathrm{TM}}$ Enzym-Immunoassay zur Bestimmung von Pneumococcus-Kapsel-Polysaccharid-(PCP)-IgG-Antikörpern. 2014; http://peramed.com/peramed/index.php?r=site/page\&view=ingUrunAgac2yii. Accessed 03.11.2016.

43. Wernette CM, Frasch CE, Madore D, et al. Enzyme-Linked Immunosorbent Assay for Quantitation of Human Antibodies to Pneumococcal Polysaccharides. Clinical and Vaccine Immunology. 2003;10(4):514-519.

44. Fishman JA, Ikle DN, Wilkinson RA. Discrepant Serological Assays for Pneumococcus in Renal Transplant Recipients. Transplant international : official journal of the European Society for Organ Transplantation. 2017.

45. Jódar L, Butler J, Carlone G, et al. Serological criteria for evaluation and licensure of new pneumococcal conjugate vaccine formulations for use in infants. Vaccine. 2003;21(23):32653272 . 